\title{
Energy and Mass Analyses of Secondary Positive Ions from Silicon Wafer Surfaces
}

\author{
Shunroku Taya* \\ (Received November 16, 1977)
}

\begin{abstract}
The kinetic energy distribution of ions liberated from a silicon wafer surface was

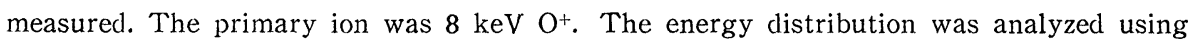
an electric toroidal sector $\left(\mathrm{r}_{e}=212 \mathrm{~mm}, \Phi_{e}=85^{\circ}, \mathrm{c}=0.5\right)$. Two new sharp energy peaks have been observed immediately below a giant sputtered ion peak.

These energetically discrete sputtered ions were analyzed with a double focusing mass spectrometer. From the results of SIMS analyses, ions of the lowest energy peak have been identified as the ambient gas ions, ions of the next higher energy peak as those from the top surface, and ions of the giant energy peak as those from the bulk.
\end{abstract}

\section{Introduction}

Recently, solid surface analyses have been risen progressively in importance in responce to the demands of semi-conductor device and metalworking industries. Although there are many solid surface analysis methods, it may be said that the most advantageous analytical method is secondary ion mass spectroscopy (SIMS) from the points of analytical sensitivity and compound ion identification.

Sputtered secondary ions have relatively broad energy compared with ions from an electron impact ion source. Therefore, it is desirable to adopt some special ion optical devices in order to analyze these sputtered ions with a high mass resolution and a high analytical sensitivity.

One ion optical device, a double focusing mass spectrometer which has radial, axial, velocity and second-order focusing properties, was examined and has been previously reported ${ }^{1)}$. Incorporating a primary ion source in this mass spectrometer, a high mass resolution SIMS instrument was realized, and a maximum resolution of 11,000 was obtained ${ }^{2)}$. A comparative study of low energy ion scattering spectroscopy (ISS) and SIMS was carried out ${ }^{3)}$ using the electric sector of the double focusing mass spectrometer as an energy analyzer.

In the mass spectra of SIMS, it is often found that ambient gas ions and solid surface contamination ions are observed 
in addition to sputtered solid bulk ions. These ion mixtures make solid surface analyses complicated. It is well known that the energy of ambient gas phase ions are relatively lower than that of sputtered bulk ions ${ }^{4-9)}$. Employing this fact, the gas phase ion mixture can be eliminated through energy selection. In this paper, experimental results of energy distribution of the sputtered ions from a silicon wafer are described using the previously reported SIMS instrument ${ }^{3)}$, and the results of SIMS analyses are reported.

\section{Instrument}

Detail of the high resolution secondary ion mass spectrometer used for the present investigation has been reported elsewhere ${ }^{1,2,3)}$. Therefore, only a brief description of the instrument will be given here. A schematic diagram of the instrument is shown in Fig. 1. It is primarily composed

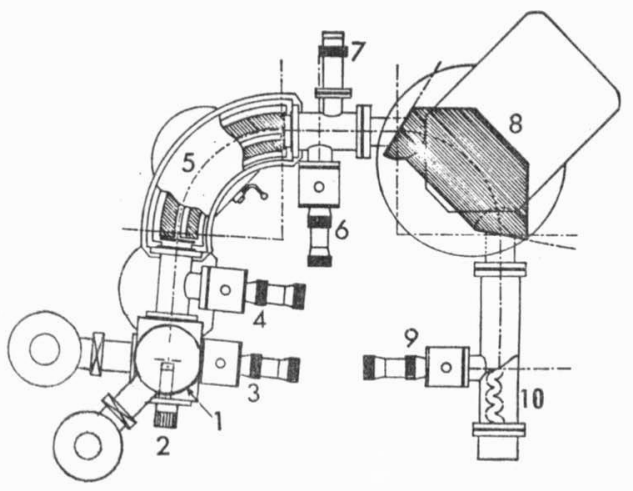

Fig. 1 Schematic diagram of the high resolution secondary ion mass spectrometer.

1. duo-plasmatron ion source, 2. sample holder, 3. variable object slit, 4 . variable $\alpha$-slit, 5. electric toroidal sector, 6. variable intermediate slit, 7. channel electron multiplier detector, 8. uniform magnet with non-zero angular entrance and exit pole edges, 9. variable collector slit, 10. electron multiplier detector. of a primary ion source, a sample chamber and a double focusing mass spectrometer.

The primary ion source is a Hitachi IMA-2 duo-plasmatron. Eight keV oxygen ions bombard a silicon wafer sample at an angle of $45^{\circ}$ to the surface plane. The sputtered secondary ions are extracted into the direction of $90^{\circ}$ with respect to the direction of the primary ion beam, and accelerated to have $2.8 \mathrm{keV}$ energy.

The kinetic energy distribution of the secondary ions is analyzed using the electric toroidal sector $\left(\mathrm{r}_{e}=212 \mathrm{~mm}, \Phi_{e}=85^{\circ}\right.$, $c=0.5$ ), and the ion current is detected by a channel electron multiplier detector at the back of the intermediate focusing point ( $\beta$-slit position). Combined a uniform magnetic sector of the non-zero angular entrance and exit $\left(\mathrm{r}_{m}=200 \mathrm{~mm}, \Phi_{m}=90^{\circ}, \varepsilon_{1}\right.$ $=32 。, \varepsilon_{2}=-10^{\circ}$ ) with the electric toroidal sector, the apparatus is used as a double focusing mass spectrometer.

The sample holder and the electric

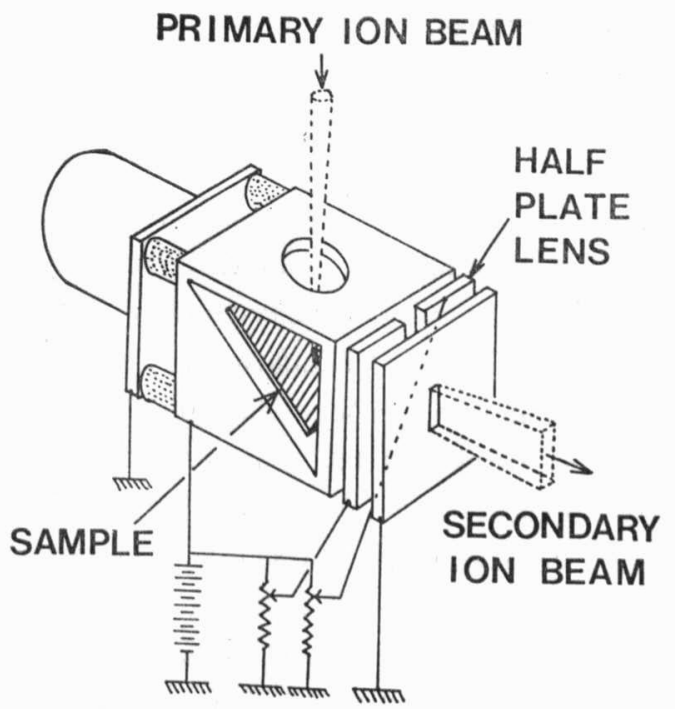

Fig. 2 Schematic diagram of the sample holder lens system. 
lens system are shown schematically in Fig. 2. The sample is covered with an equipotential box. The sputtered ions are extracted from the exit silt, and focused at the object slit position. The focus position is adjusted by a half plate lens.

Three pump systems composed of liquid nitrogen traps, oil diffusion pumps and rotary pumps are used at the ion source, sample and electric toroidal sector housings. The ultimate pressure is $2 \times 10^{-7}$ Torr. During operation, the pressure increased up to $1 \times 10^{-5}$ Torr due to the oxygen gas from the primary ion source.

\section{Experimental Results}

\subsection{Energy distribution of sputtered ions}

A silicon wafer of p-type $20 \Omega \mathrm{cm}$ was used as the sample. $8 \mathrm{keV}$ oxygen primary ions bombarded the sample surface, and the sputtered ions were accelerated to have the energy of $2.8 \mathrm{keV}$. The energy distribution of the secondary ions was measured by a rapid linear scanning of the electric sector field. One of the results is shown in Fig. 3.

The observed sputtered ions have approximately the same energy as the acceleration energy, $2.8 \mathrm{keV}$. The sputtered ion peak has a long tail on the higher energy side. While, the peak rises relatively sharply on the low energy side. A fine structure of the energy distribution could not be obtained from such a rapid scanning.

The energy distribution spectra were measured by a slow scanning of the electric field. The result is shown in Fig. 4. In this case, two sharp energy peaks, $P_{1}$ and

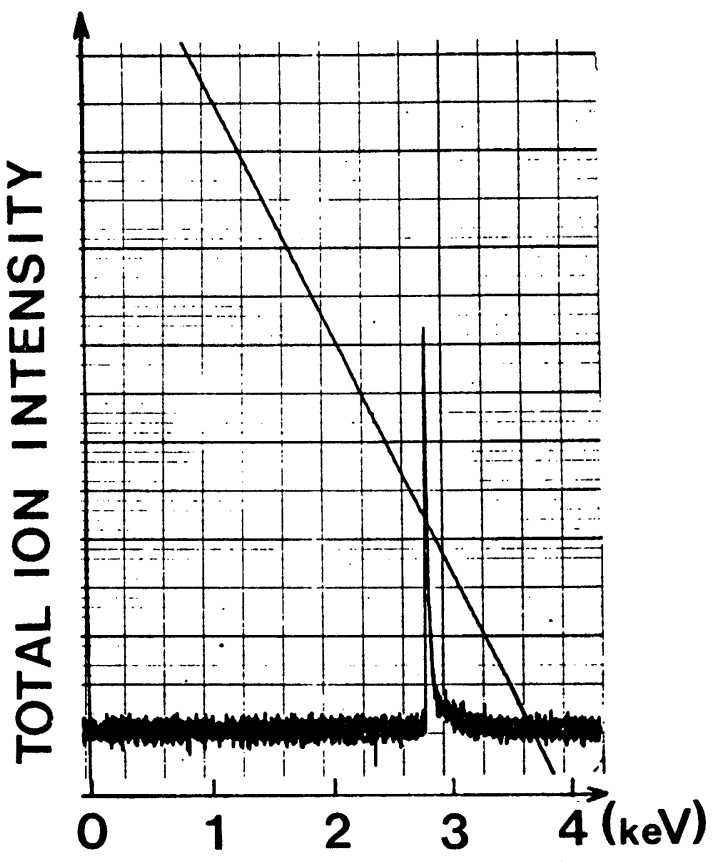

Fig. 3 Energy distribution of the accelerated sputtered ion beam. The straight line indicates the linear scanning electric sector field.

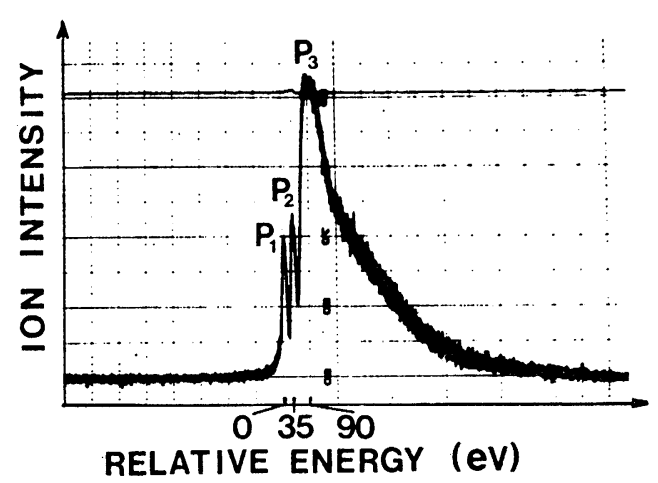

Fig. 4. Energy spectra of the accelerated sputtered ion beam.

$\mathrm{P}_{2}$, were observed on the lower side of the giant peak, $\mathrm{P}_{3}$. The upper line shows the constancy of the primary beam current. The relative energy difference between $P_{1}$ 
and $\mathrm{P}_{2}$ was measured to be about $35 \mathrm{eV}$, and the difference between $\mathrm{P}_{2}$ and $\mathrm{P}_{3}$ to be $55 \mathrm{eV}$. The actual potential of the sample surface during the ion bombardment might be somewhat different from the sample holder box due to the charge up effect. So that the energy differnce between $P_{1}$ and $\mathrm{P}_{2}$ shown here, may include the energy shift. These energy peaks were stably reproduced in several repeated measurements.

The dependence of these secondary ion energy spectra on the energy of the primary ions, $\mathrm{E}_{p}$, was examined, and the results are shown in Fig. 5. When the energy of the primary ions is increased from $6 \mathrm{keV}$ to $10 \mathrm{keV}$, the peak height of $\mathrm{P}_{3}$ is increased. However, the intensity of the two lower energy peaks, $P_{1}$ and $P_{2}$, do not change. It is found that the energy differences among the three peaks are independent of $\mathrm{E}_{p}$.

\subsection{Comparison between energy spectra and SIMS spectra}

It is interesting to identify the separated energy peaks using SIMS. The analyses were made with the double focusing mass spectrometer setting the electric sector field to the tops of the energy peaks. In this case, the channel electron multiplier detec. tor at the back of the $\beta$-slit was pulled out of the ion path.

The SIMS spectra at the lowest energy peak, $P_{1}$, are shown in Fig. 6 . The ion intensity sequence is as follows:

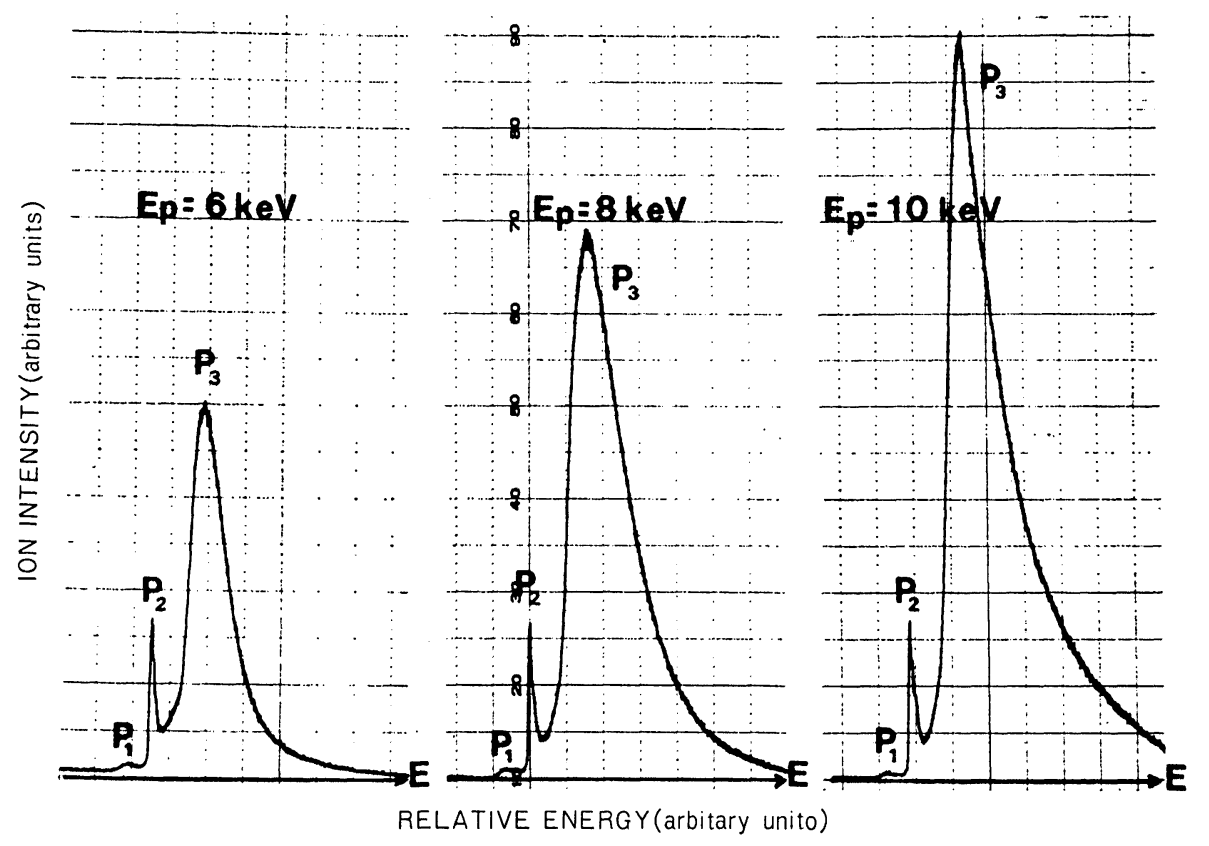

Fig. 5 Primary ion energy $\left(E_{p}\right)$ dependence of the sputtered ion energy distributions. 
Energy and Mass Analyses of Secondary Positive lons from Silicon Wafer Surfaces

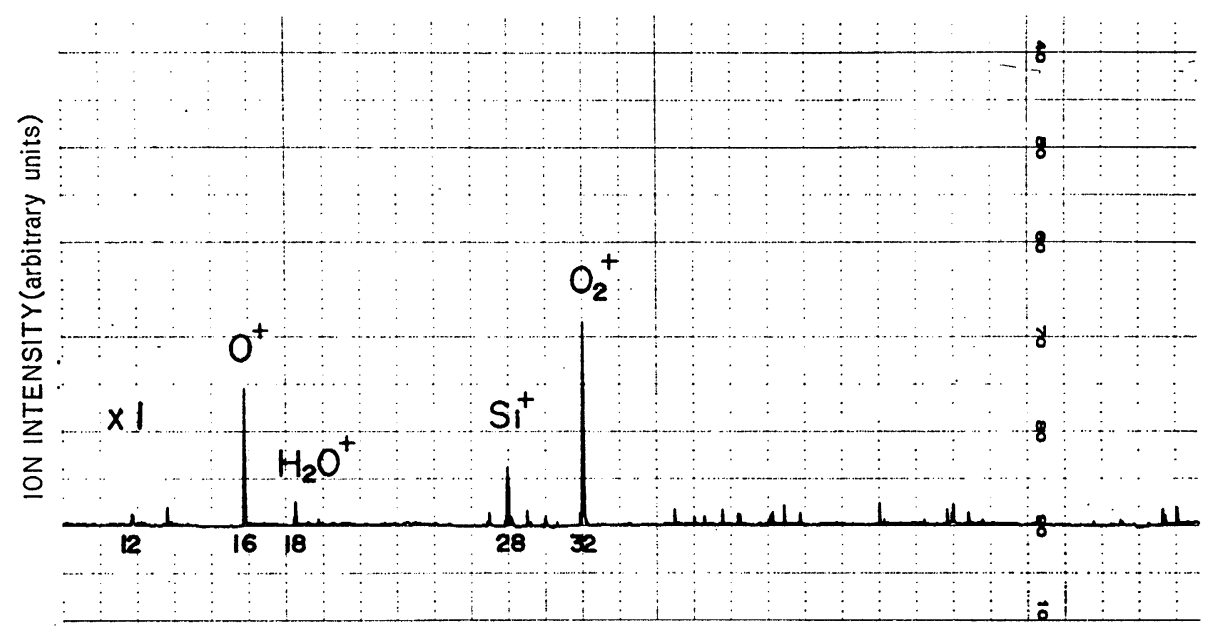

MASS NUMBER

Fig. 6 SIMS spectra for the lowest energy peak $\left(P_{1}\right)$.

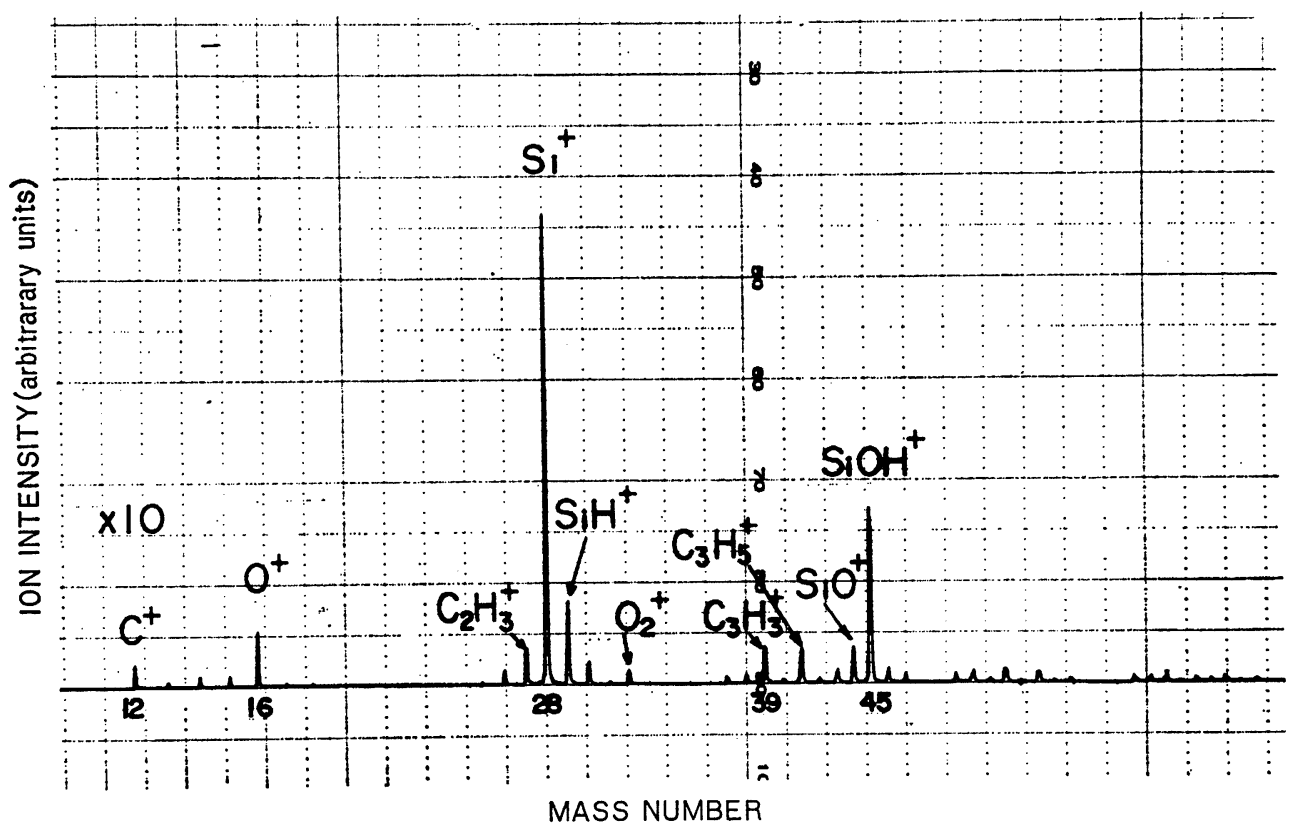

Fig. 7 SIMS spectra for the second energy peak $\left(\mathrm{P}_{2}\right)$. 


$$
\mathrm{O}_{2}+>\mathrm{O}^{+}>\mathrm{Si}^{+}>\mathrm{H}_{2} \mathrm{O}^{+}
$$

From these spectra, it is clear that the lowest energy peak, $P_{1}$, corresponds to the gas phase atoms and molecules which are ionized by the primary ions, secondary electrons and secondary ions. The oxygen is considered to be supplied from the primary ion source.

The SIMS spectra at the next higher energy peak, $\mathrm{P}_{2}$, are shown in Fig. 7. In the figure, the sensitivity of the amplifier was reduced to $1 / 10$ of that in Fig. 6 . The ion intensity sequence is as follows:

$$
\begin{aligned}
\mathrm{Si}^{+}>\mathrm{SiOH}^{+}>\mathrm{SiH}^{+}>\mathrm{O}^{+}>\mathrm{SiO}^{+}> \\
\mathrm{C}_{2} \mathrm{H}_{3}{ }^{+}>\mathrm{C}_{3} \mathrm{H}_{3}{ }^{+}>\mathrm{C}_{3} \mathrm{H}_{5}{ }^{+}>\mathrm{C}^{+} .
\end{aligned}
$$

Here, sputtered ions of silicon, silicon-com. pounds and many hydro-carbons appeared. Therefore, the second energy peak, $\mathrm{P}_{2}$, can be ascribed to the sputtered ions from the top surface of the silicon wafer.

The SIMS spectra at the giant peak, $\mathrm{P}_{3}$, are shown in Fig. 8. The sensitivity was reduced to $1 / 50$ of that in Fig. 6 . The ion intensity sequence is as follows:

$$
\begin{aligned}
\mathrm{Si}^{+}>\mathrm{SiH}^{+}>\mathrm{C}^{+}>\mathrm{CH}^{+}>\mathrm{CH}_{2}{ }^{+}> \\
\mathrm{SiO}^{+}>\mathrm{SiOH}^{+}>\mathrm{O}^{+} .
\end{aligned}
$$

As a result, it is clear that the giant peak, $\mathrm{P}_{3}$, corresponds to the sputtered ions of the silicon bulk. The observed SIMS results at the three energy peaks are summarized in Table 1. In this table, the identified ions

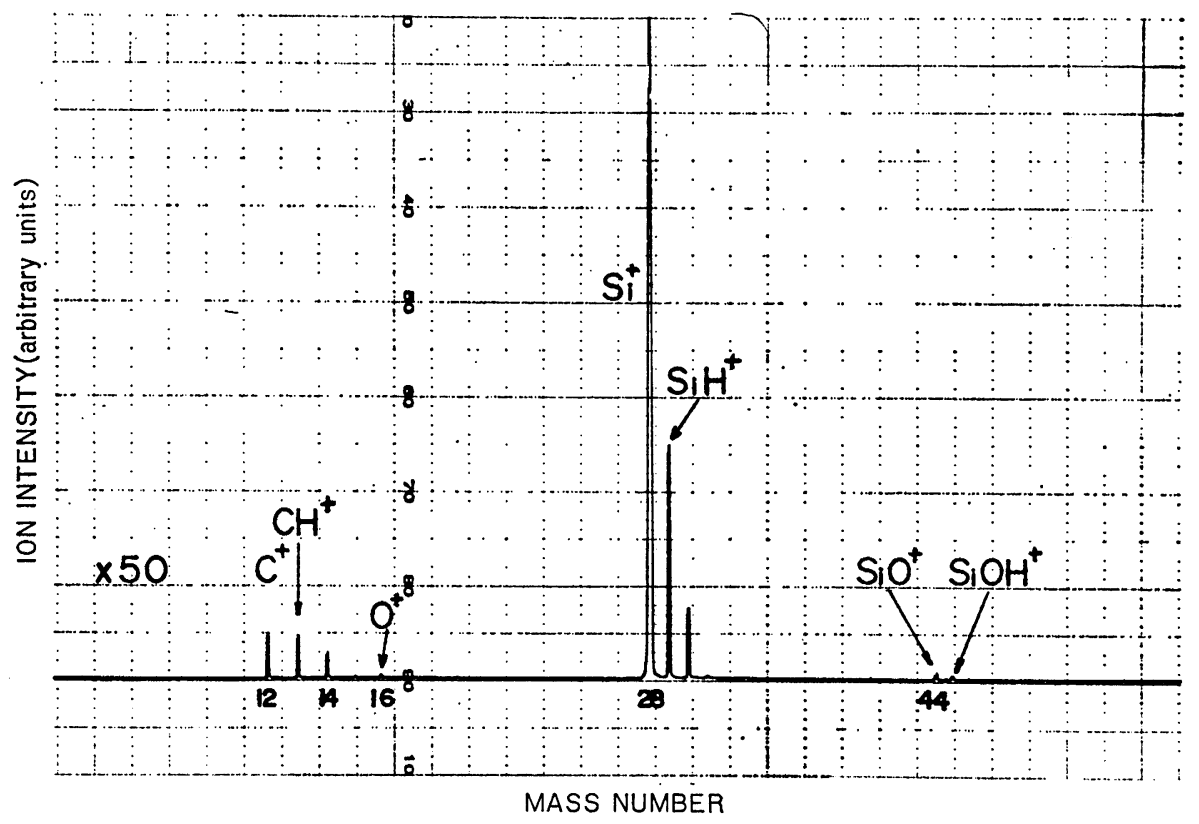

Fig. 8 SIMS spectra for the highest or giant energy peak $\left(\mathrm{P}_{3}\right)$. 
and their relative ion current intensities are given.

Table 1. SIMS analyses of three discrete energy peaks

\begin{tabular}{|c|c|c|c|c|}
\hline \multirow{2}{*}{$\begin{array}{l}\text { Mass } \\
\text { Number }\end{array}$} & \multirow{2}{*}{$\begin{array}{l}\text { Identified } \\
\text { Ion }\end{array}$} & \multicolumn{3}{|c|}{$\begin{array}{l}\text { Relative Ion Intensity for } \\
\text { Three Energy Peaks }\end{array}$} \\
\hline & & $P_{1}$ & $\mathrm{P}_{2}$ & $\mathrm{P}_{3}$ \\
\hline 12 & $\mathrm{C}^{+}$ & 0.007 & 0.15 & 1.7 \\
\hline 13 & $\mathrm{CH}^{+}$ & & 0.03 & 1.6 \\
\hline 14 & $\mathrm{CH}_{2}{ }^{+}$ & & 0.07 & 0.9 \\
\hline 15 & $\mathrm{CH}_{3}{ }^{+}$ & & 0.07 & \\
\hline 16 & $\mathrm{O}^{+}$ & 0.097 & 0.37 & 0.17 \\
\hline 17 & $\mathrm{OH}^{+}$ & & 0.01 & \\
\hline 18 & $\mathrm{H}_{2} \mathrm{O}^{+}$ & 0.017 & & \\
\hline 26 & $\mathrm{C}_{2} \mathrm{H}_{2}{ }^{+}$ & & 0.1 & \\
\hline 27 & $\mathrm{C}_{2} \mathrm{H}_{3}{ }^{+}$ & & 0.23 & \\
\hline 28 & $\mathrm{Si}^{+}$ & 0.04 & 3.1 & 100.* \\
\hline 29 & $\mathrm{SiH}^{+}$ & & 0.4 & 5.0 \\
\hline 32 & $\mathrm{O}_{2}{ }^{+}$ & 0.14 & & \\
\hline 39 & $\mathrm{C}_{3} \mathrm{H}_{3}^{+}$ & & 0.23 & \\
\hline 41 & $\mathrm{C}_{3} \mathrm{H}_{5}{ }^{+}$ & & 0.23 & \\
\hline 44 & $\mathrm{SiO}^{+}$ & & 0.3 & 0.23 \\
\hline 45 & $\mathrm{SiOH}^{+}$ & & 1.1 & 0.2 \\
\hline
\end{tabular}

* All values are normalized from this peak intensity.

\section{Conclusion}

(1) Bombarding a silicon wafer by primary $8 \mathrm{keV}$ oxygen ions, the energy distribution of the sputtered secondary ions was analyzed using an electric toroidal sector. From this experiment, two discrete energy peaks were resolved from the giant sputtered peak.

(2) Setting the electric sector field to each energy peak top, SIMS analyses were made using a double focusing mass spectr ometer. From the SIMS analyses, the following identifications were clarified:

$$
\begin{array}{ll}
\text { the lowest energy peak: } & \mathrm{O}_{2}^{+}, \mathrm{O}^{+}, \\
\text {the second energy peak: } & \mathrm{Si}^{+}, \mathrm{SiOH}^{+}, \mathrm{SiH}^{+}, \\
& \mathrm{O}^{+}, \mathrm{SiO}^{+}, \mathrm{C}_{m} \mathrm{H}_{n}^{+}, \\
\text {the highest energy peak: } & \mathrm{Si}^{+}, \mathrm{SiH}^{+} .
\end{array}
$$

(3) From the analytical results, it is concluded that the lowest energy peak corresoonds to the ambient gas ions, the second energy peak the sputtered surface ions, and the highest energy peak the sputtered bulk ions.

\section{Acknowledgement}

The author wishes to express his sincere appreciation to Professor Jun Okano of Osaka University for his valuable suggestions.

\section{References}

1) S. Taya, H. Tsuyama, I. Kanomata, T. Noda and H. Matsuda, Int. J. Mass Spectrom. Ion Phys., 26, (1978) 77.

2) S. Taya, M.Suzuki, H. Tsuyama and I. Kanomata, Int. J. Mass Spectrom. Ion Phys. in press.

3) S. Taya, H. Tsuyama, M. Itoh, and I. Kanomata, Mass Spectroscopy (Japan), 25 (1977) 251.

4) H. E. Stanton, J. Appl. Phys., 31 (1960) 678.

5) K. Nakamura, H. Tamura and T. Kondo, Japan J. Appl. Phys., 13 (1974) 917.

6) Z. Sroubek, Surface Sci., 44 (1974) 47.

7) H. Doi, I. Kanomata, and N. Sakudo, 7 th Conference on Solid State Devices, Tokyo, Japan J. Appl. Phys., 15 (1976) Suppl. 15-1, 71.

8) T. Ishitani, H. Tamura and T. Shinmiyo, Surface Sci., 55 (1976) 179.

9) M. Someno, H. Saito and M. Kobayashi, Mass Spectroscopy (Japan), 24 (1976) 173.

\section{Keywords}

SIMS

Energy distribution

Silicon wafer

Double focusing mass spectrometer

Solid surface analysis 